\title{
Long term follow up of patients with chronic pancreatitis and pancreatic stones treated with extracorporeal shock wave lithotripsy
}

\author{
H E Adamek, R Jakobs, A Buttmann, M U Adamek, A R J Schneider, J F Riemann
}

\begin{abstract}
Background-There have been conflicting reports as to whether pancreatic ductal drainage achieved by endoscopy and lithotripsy improves the clinical outcome of patients with chronic pancreatitis. Aims-To determine the clinical outcome in patients with chronic pancreatitis who received extracorporeal shock wave lithotripsy (ESWL), and were followed up for two to eight years.

Methods-Eighty patients with severe chronic pancreatitis and endoscopically unretrievable obstructive stones underwent ESWL with a piezoelectric lithotripter between 1989 and 1996. Clinical status, relief of symptoms, further endoscopic or surgical interventions, and mortality were defined.

Results-Forty three (54\%) patients were treated successfully with ESWL. The only feature associated with treatment success was the presence of a single stone rather than multiple stones. Successfully treated patients tended to experience less pain, although this did not reach statistical significance. A slight increase in weight was noted in our patients; however, there was no notable improvement in anomalous stools and diabetes mellitus. Five patients died due to extrapancreatic reasons. No pancreatic carcinomas developed.

Conclusions-ESWL associated with endoscopic drainage is a safe technique that is particularly successful in patients with a single stone. However, pancreatic drainage by endoscopy and ESWL has almost no effect on pain in chronic pancreatitis. Furthermore, endoscopic management and ESWL does not prevent or postpone the development of glandular insufficiency. (Gut 1999;45:402-405)
\end{abstract}

Keywords: chronic pancreatitis; pancreatic duct stone; extracorporeal shock wave lithotripsy; metabolic function of the pancreas

Chronic pancreatitis is an inflammatory disease leading to destruction of pancreatic parenchyma and ductal structures. In Europe, alcohol related and idiopathic pancreatitis are the most common forms of the disease. Persistent and often unbearable pain becomes the predominant symptom. Although the origin of pancreatic pain is multifactorial, ${ }^{1}$ it may be due to increased pancreatic duct pressure secondary to obstructing stones.
The techniques available for endoscopic treatment of chronic pancreatitis include sphincterotomy of the pancreatic duct and insertion of a stent. These have expanded the therapeutic spectrum that, traditionally, was based on surgical pancreatic ductal decompression. ${ }^{2-4}$ When endoscopic treatment alone fails, extracorporeal shock wave lithotripsy (ESWL) has been recommended to facilitate fragmentation and stone removal. ${ }^{5}$ Although none of these procedures has been shown to be efficacious in controlled clinical trials, they are increasingly used in clinical practice. ${ }^{67}$ Furthermore, the role of ductal decompression in preventing or postponing the development of glandular insufficiency still remains to be defined. The mean duration of follow up in most studies of endoscopic treatment has been one to two years, ${ }^{8-11}$ which is clearly too short to predict the medium term prognosis of these patients regarding the development of pain, exocrine insufficiency, steatorrhoea, insulin dependent diabetes mellitus, pancreatic carcinoma, or death.

The aim of the current study was to evaluate further the long term survival and clinical outcome of a cohort of patients with chronic pancreatitis who were treated with ESWL.

\section{Methods}

PATIENTS

Between January 1989 and July 1996, 87 patients with chronic pancreatitis were referred to our department for endoscopic treatment in combination with ESWL. We were able to make contact with $80(92 \%)$ patients in 1998 , seven being lost to follow up. The cause of pancreatitis was alcohol induced in $60(75 \%)$ and idiopathic in 20. All patients were treated with pancreatic enzyme extracts prior to entering the study. The indication for treatment was pain in all cases. Twenty one (26\%) patients were diabetic. Loss of weight had been present in $40(50 \%)$ patients for at least 12 months, and in $19(24 \%)$ patients frequent and fatty stools were evident. No previous surgery had been performed for chronic pancreatitis. All patients had main pancreatic duct stones that were not extractable by endoscopic measures (Dormia type basket or balloon tipped catheters) even after sphincterotomy of the pancreatic orifice. At endoscopic retrograde cholangiopancreatography (ERCP), multiple stones were seen in 53 patients and single stones were

Abbreviations used in this paper: ERCP, endoscopic retrograde cholangiopancreatography; ESWL, extracorporeal shock wave lithotripsy. 
present in 27 patients, together with dilatation of the proximal pancreatic duct in all patients. The diameter of the stones ranged between 5 and $15 \mathrm{~mm}$. Stones were located in the head alone in 57 patients and in the whole pancreas in 23 patients.

TREATMENT

All patients were treated in hospital. ESWL was performed with a modern second generation piezoelectric lithotripter (Piezolith 2300; Richard Wolf, Inc., Knittlingen, Germany). The system is equipped with two integrated $4 \mathrm{MHz}$ ultrasound scanners and permits ultrasound localisation of concrements. The shock waves are generated by the piezoceramic transducer and are passed into the body of the patient via degassed water. Shock wave focus energy was increased from a minimum of 600 bar to the highest tolerated energy level (maximum 1200 bar). During lithotripsy, the patient lay on the lithotripter table in a prone position. Analgesics (pethidine 50-100 $\mathrm{mg}$ or ketamine 30-100 $\mathrm{mg}$ ) and sedatives (midazolam $2-5 \mathrm{mg}$ ) were administered intravenously, if necessary. No patients were treated under general anaesthesia. A mean number of 4.1 (1-8) treatment sessions with a mean number of 14004 (3500-22 500) shock waves per patient were administered. The duration of treatment was about one hour per session.

ESWL was always followed by a further endoscopic procedure. Treatment success was defined as complete clearance of the main pancreatic duct or partial clearance that allowed implantation of a pancreatic stent.

FOLLOW UP

After participating in the ESWL trial, patients were scheduled for at least yearly follow up visits. Patients with a pancreatic stent were asked to come for routine stent exchanges after

Table 1 Clinical, radiomorphological, and treatment features

\begin{tabular}{llll}
\hline Feature & $\begin{array}{l}\text { Successful } \\
\text { treatment }(n=43)\end{array}$ & $\begin{array}{l}\text { Unsuccessful } \\
\text { treatment }(n=37)\end{array}$ & p Value \\
\hline Male sex (n) & 31 & 31 & 0.55 \\
Age (y) & $49(20-61)$ & $45(17-54)$ & 0.86 \\
Chronic alcoholism (n) & 32 & 28 & 0.94 \\
Single stone (n) & 20 & 7 & 0.03 \\
Multiple stones (n) & 23 & 30 & 0.13 \\
Stones in head alone (n) & 30 & 27 & 0.86 \\
Stones in head/body/tail (n) & 13 & 10 & 0.78 \\
Mean stone diameter (mm) & $8.6(6-15)$ & $7.9(5-15)$ & 0.75 \\
ESWL sessions/patient & $3.7(2-6)$ & $5.1(1-8)$ & 0.23 \\
Analgesia during treatment (n) & & & 0.34 \\
$\quad$ Midazolam/pethidine & 20 & 23 & 0.53 \\
$\quad$ Midazolam/ketamine & 11 & 7 & 0.41 \\
$\quad$ No analgesia & 12 & & \\
\hline
\end{tabular}

ESWL, extracorporeal shock wave lithotripsy.

Table 2 Long term analysis of clinical outcome in 80 patients with chronic pancreatitis treated with extracorporeal shock wave lithotripsy (ESWL)

\begin{tabular}{llll}
\hline Feature & $\begin{array}{l}\text { Successful } \\
\text { treatment } \\
(n=43)\end{array}$ & $\begin{array}{l}\text { Unsuccessful } \\
\text { treatment } \\
(n=37)\end{array}$ & p Value \\
\hline Considerable or complete pain relief & 34 & 27 & 0.75 \\
No further analgesia necessary & 27 & 16 & 0.23 \\
Improvement of steatorrhoea & $5 / 10$ & $4 / 9$ & 0.86 \\
Increase in body weight & $20 / 23$ & $11 / 17$ & 0.43 \\
De novo development of diabetes mellitus & 2 & 4 & 0.31 \\
Pancreatic surgery & 3 & 5 & 0.35 \\
Pancreatic carcinoma & 0 & 0 & 0.78 \\
Death & 3 & 2 & \\
\hline
\end{tabular}

three months, and later with progressively longer intervals of 6-12 months. Pain, weight, diarrhoea, presence of diabetes, and drug use were investigated. Routine laboratory tests were carried out together with an ultrasound examination. Computed tomography examinations were not performed routinely. Beginning in April 1998, attempts were made to contact all living patients or their referring physicians. A standardised questionnaire regarding pain, weight, diarrhoea, drug use, further diseases, hospitalisations, and operations was completed for all patients who were followed up. Follow up data were collected until July 1998 to obtain a minimum follow up time of two years. In the event of death before July 1998, the patient's relatives and general practitioner were interviewed and data and causes of death were recorded. As of July 1998, five patients were known to have died, and clinical details and causes of mortality were available for these patients. Seventy five patients were contacted successfully. Endoscopic re-examination was not part of the study protocol; thus a calculation about recurrent calculi cannot be made.

\section{STATISTICS}

Results are expressed as mean (SD) or as a percentage of the total number of patients. A $\chi^{2}$ analysis or the two tailed Fisher's exact test were used to compare differences between the two groups. A p value of less than 0.05 was considered to be significant.

\section{Results}

Among the 80 patients, 43 (54\%) were treated successfully with ESWL. Table 1 presents the initial clinical, demographic, and endoscopic features. Successful treatment was more frequent in patients with solitary stones (fig 1). The cause of pancreatitis, age at entry into the study, stone size and location, and treatment features were similar in the two groups. One severe bleeding episode following pancreatic duct sphincterotomy resolved with endoscopic injection therapy. Twelve patients developed transitory asymptomatic hyperamylasaemia. Bleeding into a pseudocyst occurred in a further patient. This resolved with conservative treatment.

The mean duration of follow up was 40 (2492) months. Sixty one patients showed considerable or complete relief of pain. Forty three patients did not require analgesics after ESWL. Relief or improvement of pain tended to occur more often in patients with successful ESWL treatment, but also occurred in patients whose stones persisted (table 2).

Frequent and fatty stools were present in 19 patients before treatment. At the end of follow up, symptoms improved in nine patients, whereas eight patients experienced no change. In two patients stools worsened after treatment. Long term follow up did not reveal any difference regarding the success of treatment. Comparable results were found in the 40 patients who had lost weight before ESWL. An increase in weight was observed in 31 patients. Three patients experienced no change in 

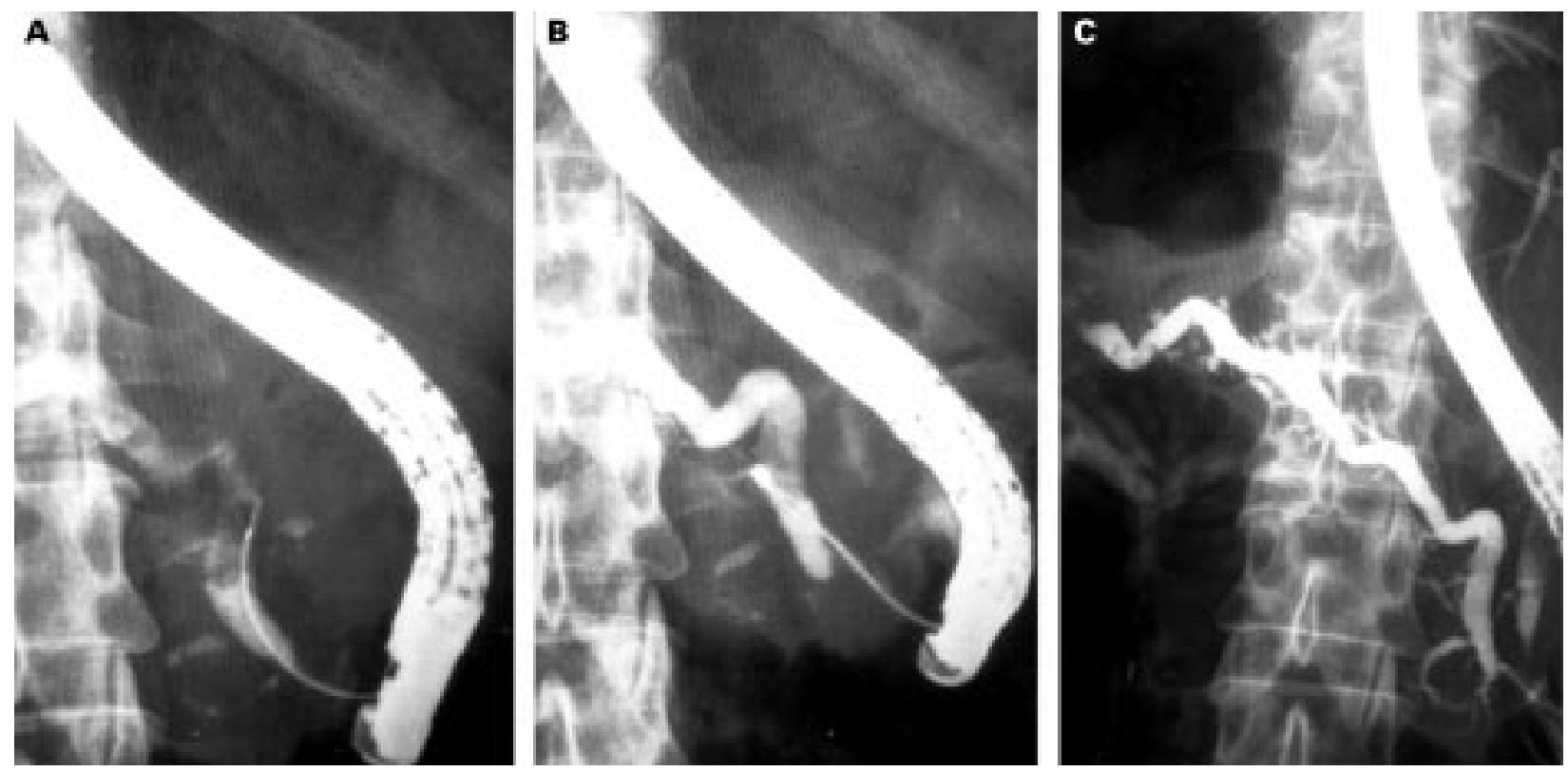

Figure 1 (A) Pancreatography shows a large intraductal concretion in a dilated main duct in the pancreatic head region. (B) After three ESWL treatments (10 000 shock waves) endoscopic basket extraction of fragments is possible. (C) Follow up pancreatogram shows complete stone removal with a subsequent decrease in pancreatic duct diameter.

weight, and six patients reported a continuous loss of weight. Preexisting diabetes mellitus was unchanged or impaired after lithotripsy; no patient stopped taking insulin and six patients developed insulin dependent diabetes mellitus after ESWL.

By the end of follow up, eight patients had undergone elective pancreatic surgery $(10 \%)$, mostly because of recurrent pain or pancreatic pseudocysts that could not be treated endoscopically. Two patients had surgery immediately after failure of endoscopic treatment. A duodenum preserving pancreatic head resection was performed in both. The other six patients underwent surgery after a mean interval of 13 (2-24) months. Two patients were operated on because of main bile duct involvement with cholangitis. Surgical treatment consisted of a pancreaticojejunostomy and hepaticojejunostomy. In all other patients, Whipple's procedure was carried out. Surgical indications and their respective frequencies showed no significant differences in relation to treatment success.

No patient developed pancreatic carcinoma. Five patients died due to extrapancreatic reasons: liver cirrhosis (one), myocardial infarction (two), colon cancer (one), lung cancer (one).

\section{Discussion}

This analysis describes long term follow up of 80 patients with chronic pancreatitis who were treated with ESWL in a clinical protocol at our institution between 1989 and 1996. ESWL is a well tolerated procedure with a low complication rate. Two major complications occurred in this series; intraduodenal haemorrhage was due to the endoscopic sphincterotomy before ESWL. Nevertheless, the majority of experts recommend pancreatic sphincterotomy before endoscopic treatment of chronic pancreatitis. ${ }^{12}{ }^{13}$ Ell et al recently reported a comparably low complication rate of $4.2 \%$ following pancreatic sphincterotomy in chronic pancreatitis. ${ }^{14} \mathrm{Up}$ to now, there has only been one larger trial of ESWL without prior pancreatic sphincterotomy. ${ }^{15}$ Together with Smits et $a l^{16}$ we emphasise that endoscopic pancreatic sphincterotomy and drainage should be undertaken by expert endoscopists at centres with special interest and experience in pancreatic disorders.

The rate of treatment success was 53\%, which is similar to that reported in other series. The only significant predictor of treatment success was the number of pancreatic stones before ESWL. Among the patients with successful ESWL treatment, single stones were more frequent than multiple stones. Published data are inconsistent. Sherman et al presented a study of 32 patients with pancreatic duct stones that were treated by means of endoscopic removal. They concluded that success of treatment was greater in patients with a small number of stones (less than three) rather than multiple calculi (more than three).${ }^{17}$ Schneider et al found that neither the number of calculi nor the stone localisation influenced the success rate in a group of 50 patients treated with ESWL. ${ }^{18}$ Furthermore, Delheye et al, who reported the largest number of patients with pancreatic duct stones treated so far by ESWL, were unable to show a statistically significant correlation between complete clearance of the main pancreatic duct and stone volume (single or multiple stones, size of the biggest stone). ${ }^{19}$ In accordance with our results, Smits et al, who focused on treatment success after endoscopic treatment in combination with intracorporeal and extracorporeal lithotripsy, also found no difference in treatment success with respect to stone location (head or body of the pancreas) ${ }^{20}$

Severe pain controllable only by analgesics is the main indication for treatment in chronic pancreatitis. The efficacy of a combination of 
ESWL and endoscopic treatment has been shown by Sauerbruch et al; $50 \%$ of their patients showed considerable or complete relief of pain. ${ }^{21}$ In contrast to our results, Sauerbruch et al found that relief or improvement of pain occurred more often in patients with complete stone clearance. Our findings are in accordance with those of Schneider et al, who also found no statistical difference in the disappearance of pain, regardless whether the pancreatic duct was cleared or whether fragments remained. ${ }^{18}$ The fact that successful treatment did not significantly influence symptomatic improvement in our group may be related to the multifactorial cause of pain in chronic pancreatitis. In addition to the elevated pressure in the pancreatic duct caused by a stone, there is also an inflammatory process in the pancreatic parenchyma and around the nerves of the pancreas.

The procedures used for evaluating faecal fats have changed over the past 10 years. Faecal fat determinations on 72 hour stool collections are quite unpopular with both patients and laboratory personnel. Other approaches such as breath tests or nuclear magnetic resonance spectrometry were not available during the first years of the study, thus the occurrence of steatorrhoea was only observed clinically. Anomalous stools were present in 19 patients before treatment $(24 \%)$. No significant changes were observed after ESWL in either group. In the 40 patients who had lost weight before ESWL, 31 reported an increase in weight irrespective of treatment success. It has been established that there is a close correlation between endocrine and exocrine dysfunction in chronic pancreatic inflammation. In our study, we were not able to improve abnormal endocrine function even in the successfully treated group of patients; indeed, six patients developed diabetes mellitus after ESWL treatment. Our results confirm former findings that, although weight gain is often observed after endoscopic treatment of patients with chronic pancreatitis, metabolic function of the pancreas is not notably improved. ${ }^{22}$ This is consistent with the observation that exocrine pancreatic dysfunction occurs many years before endocrine dysfunction. ${ }^{23}$ Cavallini et al carried out a long term follow up (mean 10 years) in more than 700 patients with chronic pancreatitis and found that at the end of the study $41.5 \%$ had diabetes. At that time, steatorrhoea was already present in $80 \%$ of patients. ${ }^{23}$

Five patients died during follow up (6\%). This low mortality rate is similar to that in other studies. ${ }^{23}$ Subsequently, mortality is known to increase, and 20 years after onset of chronic pancreatitis only $63 \%$ of patients are still alive. Our results corroborate the findings of Cavallini et al, who showed that the causes of death were mainly malignancies related to drinking and smoking, or to other diseases related to drinking habits or cardiovascular diseases. In contrast to us, and after a longer follow up, this Italian group was able to detect 14 cases of pancreatic malignancy $(8 \%) .{ }^{23}$ Our own and the reported data suggest that patients with chronic pancreatitis have a higher mortality rate than the population as a whole.
ESWL associated with endoscopic drainage is a safe technique, and its use seems very appropriate in patients with symptomatic chronic pancreatitis before proceeding with surgical interventions. However, endoscopic treatment in combination with ESWL has never been subjected to prospective controlled trials and should therefore be restricted to centres with great expertise in interventional endoscopy to include all patients into clinical trials. Long term follow up data raise doubts as to whether endoscopic management of chronic pancreatitis plays a role in preventing or postponing the development of glandular insufficiency. Consequently, the problem of calculus chronic pancreatitis has hardly been solved. For that reason, two larger and longer follow up studies of patients with chronic pancreatitis who receive endoscopic treatment are currently in progress in Germany with the aim of defining the best lifelong treatment.

1 Kloeppel G. Pathology of chronic pancreatitis and pancreatic pain. Acta Chir Scand 1990;156:261-5.

2 Banks S. Chronic pancreatitis: clinical features and medical manks. Chronic pancreatitis: clinical features and

3 Moosa AR. Surgical treatment of chronic pancreatitis: an overview. Br f Surg 1987;74:661-7.

4 Ihse I, Borch K, Larsson J. Chronic pancreatitis: results of operations for relief of pain. World F Surg 1990;14:53-8.

5 Sauerbruch T, Holl J, Sackmann M, et al. Disintegration of a pancreatic duct stone with extracorporeal shock waves in a patient with chronic pancreatitis. Endoscopy 1987;19: $207-8$.

6 Grimm H, Meyer WH, Nam VCH, et al. New modalities for treating chronic pancreatitis. Endoscopy 1989;21:70-4

7 Cremer M, Devière J, Delheye M. Stenting in severe chronic pancreatitis: results in seventy-six patients. Endoscopy 1991;23:171-6.

8 Soehendra N, Grimm H, Meyer HW, et al. Extracorporeal shock wave lithotripsy in chronic pancreatitis. Dtsch Med Shock wave lithotripsy in chro

9 Neuhaus H. Fragmentation of pancreatic stones by extracorporeal shock wave lithotripsy. Endoscopy 1991;23: $161-5$.

10 van der Hul R, Plaisier P, Jeekel J, et al. Extracorporeal shock-wave lithotripsy of pancreatic stones: immediate and long-term results. Endoscopy 1994;26:573-8.

11 Costamagna G, Gabbrielli A, Mutignani M, et al. Extracorporeal shock wave lithotripsy of pancreatic stones in chronic pancreatitis: immediate and medium-term results. Gastrointest Endosc 1997;46:231-6.

12 Kozarek RA, Ball TJ, Patterson DJ, et al. Endoscopic pancreatic duct sphincterotomy: indications, technique and analysis of results. Gastrointest Endosc 1994;40:592-8.

13 Binmoeller KF, Jue P, Seifert H, et al. Endoscopic pancreatic stent drainage in chronic pancreatitis and a dominant stricture: long-term results. Endoscopy 1995;27:638-44.

14 Ell C, Rabenstein T, Schneider HT, et al. Safety and efficacy of pancreatic sphincterotomy in chronic pancreatitis. Gastrointest Endosc 1998;48:244-9.

15 Ohara H, Hoshino M, Hayakawa T, et al. Single application extracorporeal shock wave lithotripsy is the first choice for patients with pancreatic duct stones. Am $\mathcal{F}$ Gastroenterol 1996;91:1388-94.

16 Smits ME, Badiga M, Rauws EAJ, et al. Long-term results of pancreatic stents in chronic pancreatitis. Gastrointest Endosc 1995;42:461-7.

17 Shermann S, Lehmann GA, Hawes RH, et al. Pancreatic ductal stones: frequency of successful endoscopic removal and improvement in symptoms. Gastrointest Endosc 1991; 37:511-17.

18 Schneider HT, May A, Benninger J, et al. Piezoelectric shock wave lithotripsy of pancreatic stones. Am $\mathcal{F}$ Gastroenterol wave lithotripsy of

19 Delheye M, Vandermeeren A, Baize M, et al. Extracorporeal shock-wave lithotripsy of pancreatic calculi. Gastroenterology 1992;102:610-20.

20 Smits ME, Rauws EAJ, Tytgat GNJ, et al. Endoscopic treatment of pancreatic stones in patients with chronic pancreatitis. Gastointest Endsoc 1996;43:556-60.

21 Sauerbruch T, Holl J, Sackmann M, et al. Extracoporeal lithotripsy of pancreatic stones in patients with chronic pancreatitis and pain: a prospective follow-up study. Gut 1992;33:969-72.

22 Von Tirpitz C, Glasbrenner B, Mayer D, et al. Comparison of different endocrine stimulation tests in nondiabetic patients with chronic pancreatitis. Hepatogastroenterology 1998;45:1111-16.

23 Cavallini G, Frulloni L, Pederzoli P, et al. Long-term follow-up of patients with chronic pancreatitis in Italy. Scand $\mathcal{F}$ Gastroenterol 1998;33:880-9. 